\title{
DE LA HACIENDA A LOS PUERTOS. LOS CAMINOS EN LOS PRIMEROS AÑOS DE LA REPÚBLICA DEL SALVADOR. SIGLO XVIII
}

José Ricardo Castellón Osegueda Academia Salvadoreña de la Historia ricardo.castellon@gmail.com

Recepción: 27 de enero de 2016 Aceptación: 12 de febrero de 2016 


\section{RESUMEN}

Movilidad eficiente, adecuación (mejoras y mantenimiento) e integración se plantaron desafiantes a las comunicaciones de la República del Salvador en el siglo XIX. Los retos eran mayores que las posibilidades. La disgregación vial, propia del aislamiento; los recursos limitados (agotados por las guerras federales), la visión municipalista de la responsabilidad caminera y el interés apremiante de las necesidades de la nueva configuración económica fueron determinantes en la habilitación de una red en que privó un conjunto de líneas que, saliendo de las haciendas se juntaban en puntos nodales, sacando lo producido a ferias añileras y puertos. Sobre la base de la red heredada de la época colonial, los caminos constituyeron una urdimbre vial con serios problemas para hacerse carretera y gozar de mantenimiento adecuado, las mayores preocupaciones de las autoridades en el siglo, pero la base del desarrollo vial de la era cafetalera.

\section{Palabras clave}

Integración, movilidad, mantenimiento, caminos, carreteras

\section{ABSTRACT}

Efficient mobility , adequacy ( upgrades and maintenance) and integration were planted challenging communications of the Republic of Salvador in the nineteenth century. The challenges were greater than the possibilities., The , proper insulation breakdown vial limited resources ( exhausted by federal wars ), the municipalist vision of caminera responsibility and compelling interest of the needs of the new economic configuration were key in enabling a network in which deprived a set of lines, leaving the haciendas were joined at nodal points , making the añileras produced fairs and ports. Based on the legacy network from the colonial era , the roads were a road warp serious problems to be road and enjoy proper maintenance, the major concerns of the authorities in the century, but the base of the road development of the coffee was.

\section{Keywords}

Integration, movility, maintenance, roads, highroad 


\title{
DE LA HACIENDA A LOS PUERTOS. LOS CAMINOS EN LOS PRIMEROS AÑOS DE LA REPÚBLICA DEL SALVADOR. SIGLO XVIII
}

\author{
José Ricardo Castellón Osegueda \\ Academia Salvadoreña de la Historia \\ ricardo.castellon@gmail.com
}

\section{Introducción. El desafío carretero de la República}

La República del Salvador heredó de la Colonia una básica, parcializada (variaba con los intereses productivos de las regiones) y poco o nada integradora red caminera subordinada a caminos principales y de estos a un único camino regional, ${ }^{1}$ con destino a la capital: Guatemala o a los puertos del Atlántico. Excepto cortos tramos carreteros de haciendas y poblaciones, todos eran “de herradura”. El atraso era innegable. ${ }^{2}$ Federico Estévez, Eric Magar

\footnotetext{
${ }^{1}$ María Luisa Pérez González. "Los caminos reales de América en la legislación y en la historia”. Anuario de Estudios Americanos, Tomo LVIII, I (Universidad de Sevilla; 2001): 48.

${ }^{2}$ El cruce de los ríos es un ejemplo simple. En las demarcaciones territoriales de San Salvador había seis barcas en SanVicente para cruzar el Lempa, una más en San Miguel, para remontar el río Grande, otra (de cuerda) en Gotera, una en Sensuntepeque y una más en Santa Ana. Tenían servicio de canoas San Miguel (3), Gotera (1), San Alejo (2), Sensuntepeque (3), Tejutla (10), Chalatenango (7), Santa Ana (4), Metapán (4) y Cojutepeque (1). Puentes había solo tres, uno en Opico y los otros dos en Santa Ana y Metapán (ambos de madera). El informe del Intendente de San Salvador en 1807, se acompañó de una relación de las poblaciones expresando su distancia de las cabeceras, a falta de un plano topográfico del territorio. Antonio Gutiérrez y Ulloa. Estado General de la provincia de San Salvador, Reyno de Guatemala, presentado por el intendente Antonio Gutiérrez y Ulloa en el año de 1807. Dirección General de Publicaciones, Ministerio de Educación. El Salvador, 1962. Págs. 140-145.
} 
y Guillermo Rosas, "Partisanship in nonpartisan electoral agencies and democratic compliance: Evidence from Mexico's Federal Electoral Institute,' Electoral Studies 27 (junio 2008): 257-71.

El rezago era extensivo a la región. Finalizando el siglo XVIII e iniciando el XIX, Centroamérica continuaba siendo una región con escasas posibilidades de desarrollo económico y de comunicación con el exterior. Para las provincias de San Salvador y Sonsonate, pretérito de la república salvadoreña, la prevalencia del modelo económico dependiente, tanto de la metrópoli regional, Guatemala, como de los dictámenes de la Corona y sus restricciones, había hecho perdurar el aislamiento acentuado por una situación geográfica desfavorable ${ }^{3}$ la más importante barrera para la expansión agroexportadora.

El libre comercio borbónico abrió someramente las puertas a la expansión económica sin aportar demasiado a los caminos de regiones como la de San Salvador y Sonsonate. Al mantenerse la dependencia de los grandes comerciantes guatemaltecos y sus empréstitos y continuar con el tráfico añilero por el Atlántico, estas provincias del pacífico norte debieron seguir empleando las viejas rutas regionales y las largas y difíciles rutas atlánticas, tanto terrestres como marítimas. Localmente, tampoco hubo muchas prisas por cambiar la situación. El modelo productivo añilero había probado que los caminos existentes eran tan aptos como desde hacía siglos, de manera que privó, como en otros aspectos de la vida en esta región, la elementalidad: la red resultó suficiente para permitir el flujo de lo producido a puntos nodales claves (como si de una red neuronal se tratara) y de estos a los grandes enclaves comerciales.

Con la serie de acomodos políticos derivados de la nueva era independiente, plagada en sus primeros años de pugnas, guerras y la falta de un plan concienzudo para desarollar, primero a la Federación y luego, tras su disolución en 1839, a las nuevas repúblicas, fue imposible superar un sistema

\footnotetext{
${ }^{3}$ La mayor parte del comercio internacional se efectuaba en el Atlántico y San Salvador, mientras que Sonsonate únicamente tenían salida costera al Pacífico.
} 
de transporte diseñado para ayudar a los exportadores mientras el enorme resto de la población sobrevivía o se movilizaba en torno a ese propósito. ${ }^{4}$

El presente trabajo sostiene que la economía añilera fue la dinamizadora del proyecto carretero salvadoreño en los primeros años de la nueva república, dibujando una red que sería determinante en la configuración vial de los años de la era cafetalera por venir.

\section{De las haciendas a las ferias y ciudades, de las ferias y ciudades a los puertos}

Para mediados del siglo XIX el mismo gobierno salvadoreño confesaba: "Centroamérica ha sufrido ya tres males grandes. Constitución Federal mal organizada, sistema de tratados vulnerados por tiranías locales, y el peor de todos, la dislocación y fraccionabilidad del país", 5 mediana o malmente conectado por caminos.

Había que crear una red vial para los nuevos tiempos, o adecuar la existente. Con la Independencia, poco a poco se cortó la dependencia del monopolio guatemalteco a través de medidas como la baja en los impuestos a la importación y la posibilidad de que los salvadoreños comerciaran el añil directamente con los agentes británicos presentes en Centroamérica, a través de Belice, evitando el largo traslado de la tinta a Veracruz. Adicionalmente y también como resultado de la Independencia, más barcos comenzaron a frecuentar los puertos del Pacífico, de manera que Acajutla, que solo recibía un promedio de dos barcos al año en el siglo XVIII, empezó a recibir más de diez barcos ingleses y estadounidenses en el mismo período, para intercam-

\footnotetext{
${ }^{4}$ Sin los impuestos coloniales y la ausencia de fondos gubernamentales fue limitaba cualquier iniciativa (si es que la hubo) para hacer algo por los caminos. Todo apunta a pensar que, durante este período, no se hizo ni una sola calle o puente.

${ }^{5}$ Gobierno del Estado de El Salvador, Gaceta, No. 37 (San Salvador: 3 de diciembre de 1847), 145 .
} 
biar oro, plata, productos asiáticos, vino, harina y manufacturas europeas. ${ }^{6} \mathrm{El}$ movimiento en el Pacífico se tornó tan importante que en 1824 ya habían sido habilitados los puertos de La Libertad y La Unión, ${ }^{7}$ y para 1833 cerca de la tercera parte del añil se embarcó en el Pacífico y no vía Belice. La cifra fue similar en 1839, en que casi el 28\% del añil se exportó por vía de Chile y Perú. ${ }^{8}$ Advertida la necesidad de un puerto en la bahía de Jiquilisco, se fundó, aunque de breve existencia, el puerto de La Concordia.

Estos acomodos económicos, determinaron dos medidas cruciales en términos de comunicación vial para mediados del siglo. La primera, la necesidad de reorientar los ejes nodales de comunicación, acortando distancias y rutas entre áreas generadoras y de destino de la producción agrícola y habilitando tramos carreteros. La segunda, garantizar el enlace con la comunicación marítima como prioridad. ${ }^{9}$

Que las haciendas continuaran siendo la más grande forma de propiedad y enclave productivo del siglo XIX fue un factor decisivo. ${ }^{10}$ Esto marcó el desarrollo de los territorios y determinó un primer nivel de rutas "de interconexión”. El mejor ejemplo se vio reflejado en los enclaves que apor-

\footnotetext{
${ }^{6}$ Cada año llegaban a Belice entre 70 y 100 barcos ingleses con mercadería europea y partían cargados de añil, cochinilla guatemalteca y zarzaparrilla. Los prósperos comerciantes beliceños comenzaron a cobrar una relevancia importante en Centroamérica. En El Salvador, Marshal Bennet, su mayor representante, explotó la mina del Tabanco en San Miguel. Héctor Lindo Fuentes, La Economía de El Salvador en el siglo XIX. (San Salvador: Dirección de Publicaciones e Impresos, Consejo Nacional para la Cultura y el Arte, CONCULTURA, 2002),80.

${ }^{7}$ Isidro Menéndez "Decretos de 6 de febrero y de 13 de junio de 1824 habilitando los puertos de La Libertad y La Unión” Gaceta, Tomo 2(Guatemala:1855,Recopilación de las Leyes del Salvador, en Centro-América), 229.

${ }^{8}$ Lindo Fuentes, La Economía de El Salvador en el siglo XIX, 81.

${ }^{9} \mathrm{La}$ "salida a los puertos" es una necesidad recurrente expresada en la comunicación oficial. Para el caso: Gaceta del Salvador en la República de América Central, Número 12, Tomo 3. (San Salvador: 1 de agosto de 1851), 2 y 3.

${ }^{10}$ Las haciendas continuaban contando con una infraestructura propia: "fábrica de añil y azúcar, capilla y sacristía separadas de las casas, obrajes y tanques, tanjeas, mulas y bueyes y carretas empleadas en el cultivo del añil." Gaceta del Supremo Gobierno del Estado del Salvador, No. 38, (San Salvador: 17 de diciembre de 1847),152.
} 
taron al mercado productos de particular aprecio comercial como el tabaco, la sal, la panela, el azúcar, el arroz o el hierro de Metapán, que por cierto, brindó a la localidad un desarrollo notable incluso en materia de caminos. ${ }^{11}$

El siguiente circuito de la interconexión vial fueron las ferias añileras. Eventualmente o de manera simultánea, estas ferias se acompañaban de otro dinamizador del tráfico local y regional: las ferias de ganado. A mediados del siglo XIX continuaba el tránsito de bovinos desde Nicaragua hacia Guatemala y el negocio del repasto seguía vigente en lugares como "Los Apantes" o "El Cerro", en San Julián Cacaluta, a veces para el ganado de paso a las ferias de San Fernando, Petapas y Ojo de Agua o las eventuales de El Rodeo y Esquipulas. A éstas se sumaban las ferias de Chalatenango y San Vicente, las ferias de Apastepeque, las ferias de San Miguel; y las de Santa Ana, Ahuachapán, Metapán, Jocoro, El Sauce y San Salvador. ${ }^{12}$

Estas ferias duraban entre 10 y 15 días y a veces se podían prolongar

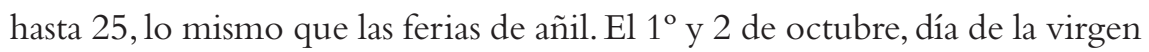
del Rosario, se celebraba en Apastepeque la famosa feria del preciado tinte. El $1^{\circ}$ de noviembre, las de San Vicente y Chalatenango, el mismo día de la fiesta de los Santos y el 21 de ese mes, la feria de Nuestra Señora de la Paz, en San Miguel. ${ }^{13}$ El 25 de noviembre se celebraba en Sensuntepeque la fiesta de Santa Bárbara y el 2 de febrero la de La Candelaria en Sonsonate.

La secuencia en el calendario festivo, dibujó un circuito de tráfico muy conveniente para el comercio. Como la feria de Chalatenango se efec-

\footnotetext{
${ }^{11}$ Gaceta del Supremo Gobierno del Estado del Salvador Número 11, Tomo 2. (San Salvador: 11 de mayo de 1849), 2.

12 Decreto de Presupuesto para los gastos de la Administración Pública en el año corriente. América Central. Gaceta del Salvador, Número 80, Tomo 7 ( San Salvador: 16 de marzo de 1859), 2. Gaceta del Supremo Gobierno del Estado del Salvador, No. 78 (San Salvador: 22 de septiembre de 1848), 319.

${ }^{13}$ Gaceta del Supremo Gobierno del Estado del Salvador, No. 78 (San Salvador: 22 de septiembre de 1848), 319.
} 
tuaba a principios de noviembre y la de San Miguel unas semanas después, los comerciantes iban de una a otra usualmente después de asistir a la feria de Apastepeque de principios de octubre y luego de participar a fines de ese mes en la de SanVicente; en espera que a últimos de noviembre e inicios de diciembre, nuevamente se dieran cita en la feria de Sensuntepeque.

A pesar de que los beneficios de las ferias variaban con el precio del añil, ${ }^{14}$ el desplazamiento comercial hizo de las ferias modos importantes de comunicación regional, llevándolas a cobrar tanta relevancia para el comercio que poco a poco se hicieron más frecuentes. Para 1859 Suchitoto solicitó se instituyera una nueva feria en esa localidad, la "feria de Santa Teresa" y en 1872 se instauró una nueva feria del Rosario (la existente era el 30 de agosto y la nueva sería el 8 de octubre) en Santa Rosa de Lima. ${ }^{15}$

Las ferias añileras, además, dinamizaban el comercio de las regiones. En ellas se daban cita pobladores comunes y comerciantes de todo tipo, desde grandes negociantes hasta buhoneros, que visitaban los lugares más distantes en una intensidad tal que el gobierno tuvo que normar su oficio en $1856 .{ }^{16}$ En la feria de la Concepción en Chapeltique, en 1881, los “comerciantes de efectos extranjeros como buhoneros y de abasto", comerciaban intensamente. ${ }^{17}$ De esta manera, la feria como evento comercial fue cambiando poco a poco el carácter de la simple fiesta patronal y la misma fisonomía de las localidades. Para 1875, por ejemplo, además de los consabidos negocios, en la fiesta de San José Villanueva, se celebrada con juegos pirotécnicos, corri-

\footnotetext{
${ }^{14}$ En 1836, por ejemplo, se decía que la feria de Ilobasco había estado "bastante desventajosa, y sin duda será á causa del precio abatido de los añiles", por lo que se esperaba que "la de hoy en Apastepeque estará mejor, y que será mas concurrida”. El Iris Salvadoreño. № 2. Fol. 5o. (San Vicente, domingo 2 de octubre de 1836), 3.

15 Boletín Oficial, $N^{\circ}$ 69, Tomo 1( América Central, República del Salvador ;San Salvador: 22 de agosto de 1872), 4.

${ }^{16}$ Cabe comentar que la feria como escenario de encuentro comercial y social alcanzó un desarrollo notable en el mundo durante el siglo XIX. El fenómeno se matizó y ganó notoriedad con los circos, exhibiciones de fenómenos y monstruos y posteriormente, juegos mecánicos.

${ }^{17}$ Diario Oficial, Tomo 10, N³7 (San Salvador, 12 de febrero de 1881),146.
} 
das de toros y hasta globos aerostáticos. ${ }^{18}$ En Nueva Guadalupe, San Vicente, se anunciaba como atractivos su "clima y aguas [...] bastante saludables sin competencia de otro lugar, la posición del pueblo y sus calles muy hermosas y estensas, lo que hace aparecer una población bastante pintoresca y sin duda alguna encontrarán los concurrentes una distraccion natural y halagüeña." En dicha feria, - se agregaba - "se venden todos los frutos del país, como frijoles, arroz, maiz, tabaco, almidon, dulce, \&,\&.”

Con los puertos como enclaves del comercio internacional, las ciudades de San Salvador (a través de La Libertad), San Miguel (a través de La Unión) y Sonsonate (a través de Acajutla) cobraron una particular relevancia como nodos de destino regional y pasos del embarque de mercancías. ${ }^{19}$ Algo similar se pretendió con Zacatecoluca y La Paz, a través del puerto de La Concordia.

Las ferias redibujaron los polos de desarrollo con las ciudades como referente. Para el caso, solo San Miguel llegó a celebrar cuatro ferias anuales que lo ponían en contacto con comerciantes de Honduras y Nicaragua, ${ }^{20}$ además de más distantes destinos a través del puerto de La Unión. Incluso en San Salvador, el presidente Barrios decretó, en 1861, el traslado de la celebración de la fiesta del Salvador del Mundo al 25 de diciembre. Los beneficios, se decía, "además de una estación más seca y fresca, serían que, habiendo pasado las importantes ferias de Chalatenango, San Vicente, San Miguel y Sensuntepeque, regresan en ese tiempo los comerciantes de la capital, Santa Ana y Guatemala y reunidos en San Salvador podrán efectuar una última transacción, además de que en la fiesta del Salvador del Mundo siempre se

\footnotetext{
${ }^{18}$ Diario Oficial del Salvador en Centroamérica, № 247, Año 1 (San Salvador, 3 de noviembre de 1875), 4.

${ }^{19}$ Unas décadas más tarde tocaría el turno a Santa Ana, como consecuencia del despunte cafetalero, buscar la salida al mar por el puerto de Acajutla, aunque el mayor beneficio vino del ferrocarril.

${ }^{20}$ Gaceta del Supremo Gobierno del Estado del Salvador, N58 (San Salvador: 5 de mayo de 1848), 229. En 1867 se instituyó en San Miguel, por ejemplo, la fiesta de la villa de San Carlos "como feria comercial por su crecimiento de población y añiles".
} 
reúnen algunas partidas de añil, que con facilidad pueden ser transportadas al puerto de La Libertad," así como otras mercaderías "lucrativas" como ganados, quesos, azúcar, panela o café. ${ }^{21}$

No se dudaba del impulso que ya tomaba la agricultura y el comercio de alimentos salvadoreños dada la necesidad de los mismos para la navegación, como ya sucedía con los altos precios a que se vendían los productos en La Unión, que había visto incrementar su movimiento hasta que recibirá, para el siglo XIX, la mayor parte de las importaciones del Estado. ${ }^{22}$

\section{ILUSTRACIÓN 1}

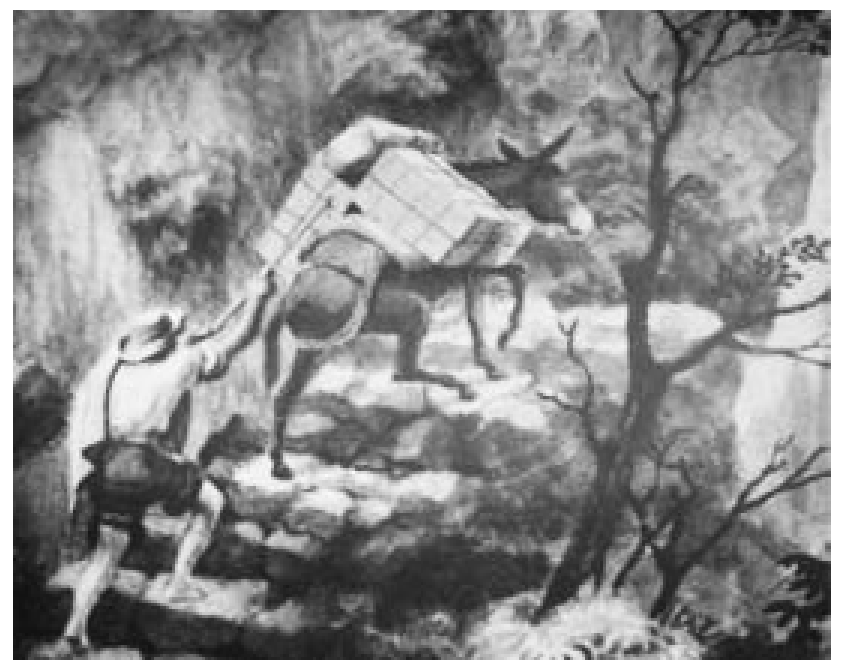

La mula fue el medio de transporte por excelencia en la época colonial

\footnotetext{
${ }^{21}$ República del Salvador. América Central. Gaceta Oficial, No 4, Tomo 10 (San Salvador: 26 de octubre de 1861), 2.

${ }^{22}$ Gaceta del Supremo Gobierno del Estado del Salvador No 3 (San Salvador: 16 de abril de 1847), 14 .
} 
Se esperaba que todo esto evidenciara la importancia de los caminos y puertos y las mejoras que en ellos debían hacerse, ${ }^{23}$ lo que sin duda sucedió. Para 1848 se hizo carretero el camino entre San Miguel y su puerto. ${ }^{24}$ Similares esfuerzos se hicieron en el camino de Sonsonate a Acajutla, en cuyo radio se consideraba vital la conducción de las aguas del río Ceniza, que separaba Izalco de Sonsonate, debido a que sus recurrentes crecidas dañaban o derrivaban sus puentes, el último de los cuales recién se había estrenado en $1845 .^{25}$ Para junio de 1849 continuaba la construcción de la carretera al puerto de La Libertad. Respecto de La Concordia, ese mismo año se hicieron esfuerzos por llevar el comercio a este puerto y facilitar el transporte desde él a la ciudad de SanVicente a través de un camino que también se haría carretero. Saliendo de Zacatecoluca se llegaba en cinco horas al puerto de La Concordia (también conocido anteriormente como Jaltepeque), ${ }^{26}$ pero ya se decía no solo que en el puerto había "una estensa casa aduana suficiente para recibir los cargamentos" que se desembarcaban, sino que además se contaba con los "alijos que para ello se necesitan".

Los resultados no se hicieron esperar. Con las mejoras al camino de San Salvador a La Libertad fue posible trasladar a San Savador todas las mercaderías extranjeras que a inicios de los años cincuenta, llegaron en el primer buque; todas en carretas. Los beneficios de un camino carretero conectando al Puerto de La Libertad con la ciudad más grande del Estado auguraban para muchos notables exportaciones de añil, pero también de azúcar, maíz y

\footnotetext{
${ }^{23}$ Gaceta del Supremo Gobierno del Estado del Salvador Nº 6, Tomo 2 ( San Salvador: 6 de abril de 1849), 2.

${ }^{24}$ Gaceta del Supremo Gobierno del Estado del Salvador, №41 (San Salvador: 3 de enero de 1848), 161.

${ }^{25}$ El Salvador Rejenerado, T.1, N³4. (San Salvador: 5 de agosto de 1846), 356.

${ }^{26} \mathrm{El}$ puerto quedaba a siete leguas de Zacatecoluca, 10 de SanVicente y Cojutepeque y 15 de la capital. "Todo el tránsito puede hacerse de rueda escepto el último". Gaceta del Gobierno del Salvador en la República de Centro América, N¹9, Tomo 4 (San Salvador: 5 de agosto de 1853), 2 y 3.
} 
otros productos a Panamá y California. ${ }^{27}$ En un país donde los mejores caminos apenas podían diferenciarse de los llamados "caminos de ganado", según palabras de extranjeros, esto era ciertamente importante. ${ }^{28}$ Pianos, mármoles y otros muebles pesados fueron conducidos a San Salvador en carretas, de las que ya había cantidad suficiente un mes después de iniciadas las operaciones del puerto. Los bajos costos de los fletes eran contrastados con los de otros puertos. En 1852 el flete de La Libertad a San Salvador era de un real por arroba y mientras la conducción de un piano que de Acajutla o La Unión iba a San Salvador costaba de 60 a 100 pesos o más, por La Libertad costaba solo cinco. $^{29}$

En sentido inverso, los enclaves portuarios poco a poco se transformaron en poblados y se pretendía una sinergia entre crecimiento de la agricultura y aproximación de los frutos a los puntos de embarque. En las inmediaciones del puerto de La Libertad se sembraba mucho maíz, arroz y otros productos para exportarlos a Panamá o California, ${ }^{30}$ y se advertía la posibilidad de cultivar añil y cosechar bálsamo en la región colindante, así como algodón a gran escala, abarcando las más de 30 leguas que habían entre el puerto de La Libertad y la bahía de Conchagua, ${ }^{31}$ considerando que ya para 1852, en las inmediaciones de la bahía de Jiquilisco y del puerto de La Libertad se cultivaba algodón con excelentes resultados. En La Unión, la construcción del muelle se acompañó en 1854 de una serie de obras, fundamentalmente de empedrados, en la población y el año anterior se había

\footnotetext{
${ }^{27}$ Gaceta del Salvador en la República de Centro América, № 4, Tomo 3 (San Salvador: 14 de mayo de 1852), 2.

${ }^{28}$ Ephraim G. Squier. Apuntamientos sobre Centroamérica. Honduras y El Salvador. (Traducción de León Alvarado. Notas actualizadas de William V. Davidson). Colección Cultural de Centroamérica Serie Viajeros N5.(Managua: Fundación Vida, 2004), 326.

${ }^{29}$ Gaceta del Salvador en la República de Centro América, N 54, Tomo 3 (San Salvador: 9 de julio de 1852), 2.

${ }^{30}$ Gaceta del Salvador en la República de Centro América, N46, Tomo3 (San Salvador: 7 de mayo de 1852), 3.

${ }^{31}$ Gaceta del Supremo Gobierno del Estado del Salvador, Nº 4 y 6 (San Salvador: 16 y 30 de abril de 1847), 13 y 24.
} 
abierto la calle de El Calvario, con 400 varas de sur a norte, en "una obra costosa pues mucha piedra grande estaba enterrada, haciéndose necesario emplear taladro y pólvora". Lo mismo se hizo en Acajutla, donde para 1854 se trabajó en varios empedrados.

\section{El esfuerzo por la vía carretera}

Hasta la Independencia, los tramos carreteros estaban restringidos a la esfera local y a algunos tramos de las regiones. Para 1807, la categorización que orientaba su utilidad era simple: "fuera de camino real" y "de camino real", también llamados eventualmente "de provincia”. Estos últimos, los más importantes, garantizaban en la América colonial que el modelo económico del Estado se desarrollara de manera fluida y sin trabas. ${ }^{32}$ Pero aun con su acusada importancia, estos caminos no eran una vía uniforme ni técnicamente completa, debido a que dependían en su habilitación y mantenimiento de los hacendados y los alcaldes. Tramos desiguales en calidad eran lo común. Por lo demás, tampoco eran rutas únicas, pues la falta de mantenimiento obligaba tener presentes rutas alternativas a su recorrido, frecuentemente interrumpidas por el clima o los accidentes geográficos.

En la concepción vial el medio hace el camino. Para inicios del siglo XIX la red vial se mostraba suficiente para el paso del principal medio de transporte: el ganado equino. El caballo era común en Centroamérica, pero su uso estaba limitado a los puertos para tirar carros, así como para ser montados por privilegiados jinetes. Eran más importantes los bueyes para la siembra, el trapiche, tirar carretas en haciendas, ciudades y tramos de caminos. Pero para el transporte a distancia la reina fue la mula, que podía cargar hasta 300 libras si se toma como referencia los dos zurrones de añil que cargaban, uno a cada lado, mientras viajaban en patachos de hasta 50 animales juntos.

\footnotetext{
${ }^{32}$ En América es posible hacer una distinción simple de los caminos reales a la llegada del siglo XIX. Unos, generalmente largos, con una visión evidentemente integradora del territorio y otros, más cortos, empleados como vías de comunicación imprescindibles para el tráfico fundamental.
} 


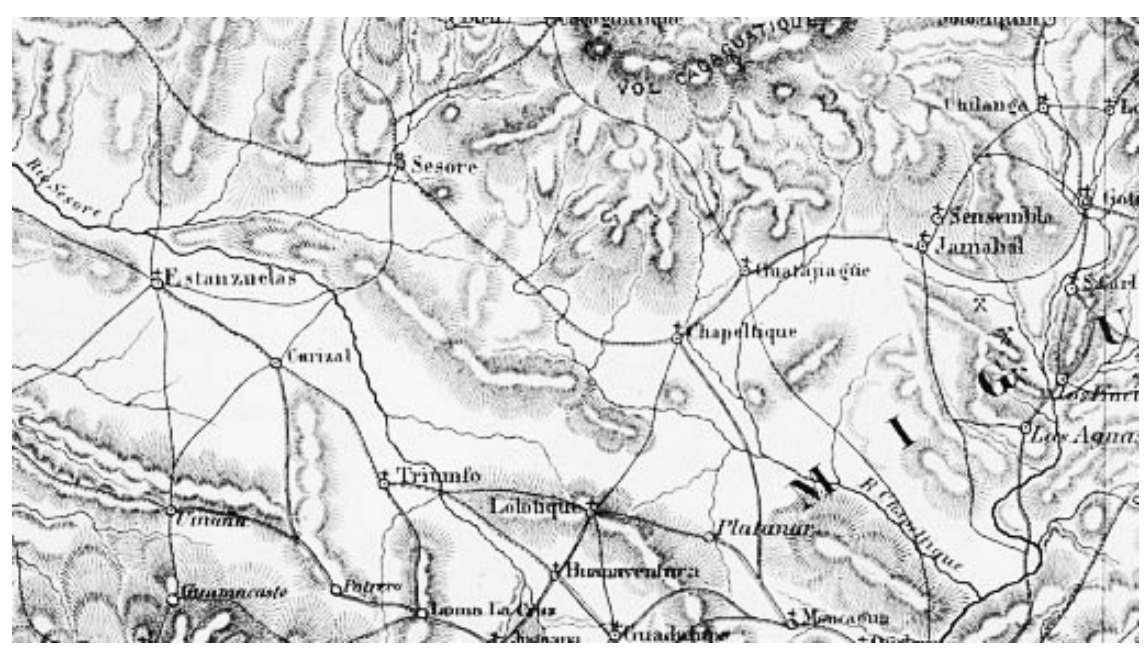

Una red neural.

(M. Sonnenstern, primer mapa de la República del Salvador, 1858)

Solo la necesidad de trasladar más volúmenes de producción hizo que la carreta cobrara relevancia en el nuevo período, orientando subordinando a la rueda los esfuerzos viales. Hubo resistencias, por ejemplo, de los dueños de patachos de mulas, pero en 1849 se hacían expresos los propósitos: "Cuando tengamos pues, caminos de ruedas, á los puertos del pacifico, y que estos tengan así mismo mejoras y comodidades para el comercio, podremos estraer aun aquellos artículos que por su poco valor no sufren gravamen alguno y encontraremos un Mercado mas barato para nuestro consumo"33. Nuevamente los beneficios se evidenciaron con los hechos: solo con la apertura del camino carretero de San Miguel al puerto de La Unión, en 1848, se esperaba reducir el valor del flete un 50\%, ahorrando adicionalmente el tiempo y gasto que tomaba deshacer y hacer la carga, lo que hacía que el viaje tomara dos

\footnotetext{
33 Gaceta del Supremo Gobierno del Estado del Salvador, № 1, Tomo 2 (San Salvador: 2 de marzo de 1849), 1 y 2.
} 
días, eso, gracias además a la abundancia de carretas. "Las grandes sumas que el comercio pagaba de fletes, la ganaban antes del camino, solo el individuo que podia tener un valor considerable en mulas". ${ }^{34}$

Sin embargo, el panorama se presentaba complejo: las vías solo eran uno de los problemas a resolver, su construcción estaba en manos de pobres municipios y su mantenimiento era un desafio avasallador.

Huelga decir que los primeros años del establecimiento de la república salvadoreña estuvieron plagados de necesidades. Además de la fábrica y reparación de cabildos e iglesias, también estaban la habilitación de escuelas y el pago a sus maestros, la persecusión de los delincuentes, la organización de grupos para combatir las plagas y pestes o más tarde, la introducción de agua en las localidades, por mencionar las mayores. Las dificultades venían de que los gastos crecían, más no los ingresos. La inversión en obras públicas en La Paz, en abril de 1857 fue reveladora: aunque el cargo (ingresos) fue de 910.7 1/2 pesos y el egreso (data) de 250.4, de estos sólo 19.2 pesos fueron dedicados a obras públicas, en tanto que 150.4 1/2 fueron usados para pagar el sueldo de los empleados. ${ }^{35}$ En otros ejemplos, ese mismo año, sólo la reparación de las fuentes públicas de Santa Ana requirió la mitad de los productos anuales.

La responsabilidad del mantenimiento vial había sido delegada desde la Colonia a los propietarios privados, ${ }^{36}$ y a los municipios. La Corona, por su parte, fiel a la práctica de no emplear caudales de la Real Hacienda, siguió el lema de dejar hacer, dejar pasar, correspondiendo a los vasallos lograr el tránsito. ${ }^{37}$ La situación se prolongó después de la Independencia. Desde la Recopilación de Leyes de la República de 1855 hasta el fin del siglo XIX se

\footnotetext{
${ }^{34}$ Gaceta del Supremo Gobierno del Estado del Salvador, No 41 (San Salvador: 3 de enero de 1848), 161.

${ }^{35}$ Gaceta del Salvador, N¹5, Tomo 6 (Cojutepeque, 13 de junio de 1857), 1.

${ }^{36} \mathrm{El} \mathrm{mal} \mathrm{endémico} \mathrm{de} \mathrm{los} \mathrm{caminos} \mathrm{coloniales} \mathrm{es} \mathrm{que} \mathrm{fueron} \mathrm{objeto} \mathrm{de} \mathrm{abusos} \mathrm{para} \mathrm{fines}$ particulares.

${ }^{37}$ María Luisa Pérez González, "Los caminos reales de América en la legislación y en la historia”, Anuario de Estudios Americanos, Tomo LVIII, I, (Universidad de Sevilla, 2001), 47.
} 
estableció básicamente que los habitantes del Estado debían trabajar dos días al año en los caminos de un pueblo a otro. Para ello, los alcaldes levantarían lista de los hombres capaces y donde el camino pasare, concertar las mejoras. También las alcaldías debían velar por los puentes y su estado, así como la ampliación de pasos angostos. ${ }^{38}$ Por lo demás, se creó una contribución que iba al Fondo de Caminos, que los municipios también se encargaban de recaudar pero que eran canalizados al gobierno central.

Algunos autores no dudan en afirmar que los altos costos del mantenimiento de los caminos se debieron principalmente a la escasez de mano de obra, sumada a la inestabilidad política hasta casi el fin del siglo. ${ }^{39} \mathrm{El}$ punto crítico de la disponibilidad de brazos era que ya de por sí hacían falta para sacar las cosechas añileras, ${ }^{40}$ una circunstancia que se agravaba con el sacrificio de la producción alimenticia por la producción agroexportadora. El problema real de las haciendas municipales fue la existencia de una estructura de recursos fiscales -al igual que en el plano estatal-, que en lugar de servir para atender a las necesidades de la población, estaba diseñada a partir de principios en los que primaban los intereses de los "propietarios", es decir, no afectar a su propiedad -inmobiliaria o empresarial-, ni a sus exportaciones, ni a sus rentas. Cuando se expropiaron los ejidos la situación no cambió, pues el problema no era el canon de ejidos o su desaparición, sino la inexistencia de

\footnotetext{
38 Isidro Menéndez Gaceta, Tomo 2, Libro 4º , título 14 (Guatemala:1855,Recopilación de las Leyes del Salvador, en Centro-América), 255-258.

${ }^{39}$ Lindo Fuentes, 132.

${ }^{40}$ Para 1854 en Cinquera, nada se ha hecho "á causa de la mucha escasez y porque sus vecinos se han ocupado en sus temporadas". Mientras, en Azacualpa, Chalatenango, no se ha hecho ninguna obra pública pues sus habitantes "se han ocupado en la elaboración del añil”, lo mismo que en San Vicente, donde "en vista de hallarse la mayor parte de trabajadores ocupados en sembrar tunalmiles" y "en la elaboracion de las cosechas de añil" se ha hecho "muy pocos trabajos publicos".
} 
un canon sobre la propiedad rural, o su producción, que gravara proporcionalmente a los propietarios, especialmente a los hacendados. ${ }^{41}$

En tal contexto, los esfuerzos camineros se concentraron en el elemental principio de mantenerlos transitables. El gobierno central, por su parte, se ocupaba de recordar la necesidad de que los caminos "se compongan forzosamente en su totalidad dos veces en el año, sin perjuicio, se entiende, de todos aquellos reparos que las circunstancias vayan demandando para la comodidad del tráfico". ${ }^{42}$

La mayor dificultad siempre fue solventar las irregularidades del terreno, la vegetación y el clima. Las primeras afectaban definitivamente las vías, que evitaban los accidentes geográficos alargando las rutas, sin por ello ser menos riesgosas; la vegetación, por su parte, crecía rápidamente e invadía en todo el sentido de la palabra las rutas. Respecto del clima, las lluvias derivaban en los más variados daños y catástrofes. Era usual que se desbordaran ríos sin que nadie pudiera hacer nada más que lamentar los daños derivados. Por causa de inundaciones se debían hacer grandes rodeos. A estos fenómenos hubo que sumar la recurrencia de los terremotos (solo en el siglo XIX se contaron cerca de 15) y las erupciones volcánicas. ${ }^{43}$ Los embates de la naturaleza llegaron a justificar, aunque fuera eventualmente, las faltas a la obligación del mantenimiento de los caminos por parte de los municipios.

\footnotetext{
${ }^{41}$ Antonio Acosta y María Julia Flores Montalvo, "Municipio y Estado: La política municipal de los gobiernos de El Salvador hacia 1900", Carlos Gregorio López Bernal (compilador), Poder, actores sociales y conflictividad. El Salvador, 1786-1972. (San Salvador: Dirección Nacional de Investigaciones en Cultura y Arte de la Secretaría de Cultura de la Presidencia, San Salvador, 2011), 191.

${ }^{42}$ Gaceta del Gobierno del Salvador en la República de Centro América, № 64, Tomo 4 (Cojutepeque: 21 de julio de 1854), 3.

${ }^{43}$ La erupción del volcán de Izalco, en 1859, causó daños en la villa y poblados de la zona, destruyendo sus iglesias, cabildos y casas de teja (las de "los principales" de aquellos vecindarios), pero además, calles y agrietando el puente sobre el río Chutil, uno de los pocos con que contaba la República.
} 
Las mejores condiciones para resolver los problemas de transitabilidad las presentaba el verano, pues el invierno que tanto afectaba a los caminos, también afectaba su reparación. Sin embargo, el problema era recurrente. Aunque en buenas condiciones algunos caminos permitían el paso de carretas a veces cargadas con importante y escasa maquinaria (en 1866, en el camino de Sonsonate a Acajutla, se transportó en una ocasión dos máquinas de vapor), con el siguiente invierno, se volvían a estropear. Ese mantenimiento volvía a reclamar recursos y esfuerzos.

Ese denuedo era diezmado por otros males como las plagas y la escasez. En 1854, mientras en Olocuilta se informaba que las obras públicas se habían paralizado por la copiosidad de las aguas, de Talpa se informaba que el atraso se había debido a que sus pobladores estaban ocupados combatiendo al chapulín saltón. Ni siquiera se había podido reparar el camino al puerto de La Concordia. Los otros municipios, se decía, "no hacen más que repetir el informe anterior argumentando escasez de víveres y de fondos algunos de ellos". De San Vicente se informaba que siendo el mes de julio de penalidades y angustias no se había podido obligar a los trabajadores a cumplir su deber para aportar fondos. Apenas en la cabecera se habían hecho algunas calzadas de madera en calles de barrios que las necesitaban, mientras "se trabaja en la composicion del camino que por la costa va a San Miguel". En 1854 en Verapaz, un nuevo camino se abrió con la participación de ocho a diez hombres, pero se aclaraba que eran los menos ocupados en sus casas o siembras. En ambos departamentos apenas se había hecho alguna reparación en 1854 por causa de que "sus habitantes apenas subsisten", mientras que en Cojutepeque, la entonces capital, las avenidas y calles continuaban en lamentable abandono con el monte creciendo por todas partes en las calles secundarias y con accesos rodeados por precipios que requerían atención. ${ }^{44}$

\footnotetext{
${ }^{44}$ A pesar de que para 1855 Cojutepeque fungió como capital de la República, no se advierte una preocupación o esmero particular por sus caminos. A lo mejor el período fue muy corto y otras preocupaciones ocupaban la mente de las autoridades.
} 
En el mismo informe de gobernadores de ese año, otros pueblos de Sonsonate, con excepción de Ahuachapán y Guaymoco, no daban cuenta de haber hecho obra de reparación caminera alguna por causa de lo copioso de las lluvias y la escasez de víveres, así como la obra destructora del chapulín saltón. ${ }^{45}$ Las mismas dificultades eran expuestas en departamentos como San Salvador y Chalatenango.

Sin duda, estos males se agravaban con la ausencia de técnicas carreteras adecuadas. Si ya los inviernos evidenciaban la necesidad de obras como puentes, diques, calzadas o muros, también demostraban la ausencia de una tecnología tan simple como eficientes sistemas de conducción del agua, cuya acción hacía zanjas anegadas y de difícil paso, como la legendaria zanja y cuesta del Guarumal. Más sencillo resultaba empedrar los pasos fangosos, dándose prioridad a poblados importantes, ciudades y tramos de caminos (sobre todo si eran reales), particularmente cuestas y planicies. Las elementales herramientas eran caras y escasas y aun así, se criticaba las reparaciones, hechas con desidia o ignorancia: en el camino que pasaba por "los pantanos del valle de San Antonio" - decía en 1858 el gobernador de San Miguel- se había colocado una gran cantidad del mejor material sobre el suelo anegado "sin un cimiento sólido que pudiera aguantar el peso del material y de la comunicación; asi es que ahora este camino es muy dificil de pasarse, las piedras entremetidas en el fango impiden el tránsito mas bien que favorecerlo". ${ }^{46}$ En 1857 se informaba que las vías de comunicación en el departamento de San Salvador no estaban en el mejor estado, sobre todo por causa del invierno: "las fuertes corrientes causadas por la copiosidad de las lluvias los trabajan y desmejoran de un dia para otro, con tanta mayor facilidad, cuanto que los terrenos son por naturaleza flojos y deleznables y porque, por otra parte, ya por falta de conocimientos o de recursos en las pequeñas poblaciones, no se componen los malos pasos con la solidez y formalidad necesarias".

\footnotetext{
45 Gaceta del Gobierno del Salvador en la República de Centro América, No 66, Tomo 4 (Cojutepeque: 4 de agosto de 1854), 3.

${ }^{46}$ Gaceta del Gobierno del Salvador en la República de Centro América, No 53, Tomo 6 (Cojutepeque: 28 de octubre de 1857), 4.
} 
Caminos donde corregir la amplitud era muy difiicil, eran aprovechados por malechores que asaltaban a los viajantes. Pero ésta era solo una de las dificultades más bien secundarias, relacionadas con las mínimas condiciones para el transporte de personas. La constante fue la ausencia de posadas y cuando las hubo, que reunieran las mínimas condiciones de salubridad.

\section{El esfuerzo caminero y la incorporación de mejores técnicas constructivas}

Se buscó solventar las necesidades de diversas maneras. Los municipios procuraron mejorar su recaudación de fondos, se buscaron maneras de contar con más mano de obra mediante el empleo de ociosos y ebrios, así como de reos; se normó la recaudación del "Fondo de Caminos" y se pretendió una mejor distribución del mismo desde el gobierno central, así como de su administración a través de Juntas locales (que eventualmente también recaudaban fondos para proyectos específicos). Finalmente, se contó con una mayor incidencia gubernamental compartida con el capital privado.

De esta manera, pudo hacerse carretero el camino entre San Salvador y el Puerto de La Libertad, o introducirse mejoras más duraderas en caminos como el de San Miguel a La Unión o Sonsonate a Acajutla. Sin embargo, la habilitación carretera fluctuó entre las emocionadas aperturas y las urgentes reparaciones debido a las insuficientes reparaciones y la deficiente tecnología caminera. Las técnicas de ingeniería eran sumamente simples. Para hacer calzadas y rellenar socavaciones se acopiaba piedras, también usadas en empedrados, reparación de puentes, ampliaciones en los estrechos, desagües, rellenos, reparación de lugares fangosos o callejones. Aunque las transformaciones a los trazos tuvieron más vigencia con la creación de caminos carreteros, también se varió el trazo de algunos caminos antiguos y nuevos, no carreteros. La práctica sin embargo, tenía más un propósito funcional, pues las correcciones se introdujeron cuando fue necesario salvar dificultades como zanjones o quebradas. 


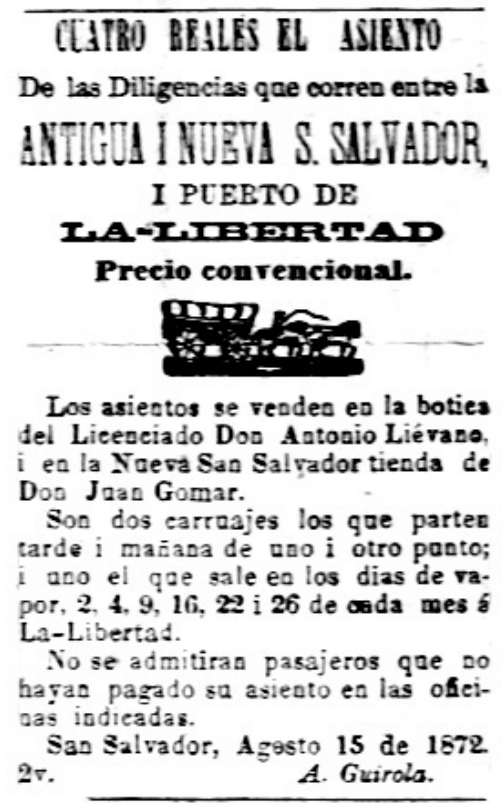

Anuncio publicado en el Diario Oficial, 1872.

En justo reconocer, a falta de recursos, el ingenio del que los ejecutores debieron echar mano. Se desmontaba usando machetes, se reparaba empleando almádanas, palas y azadones. A falta de ingenieros se ejercitaba el ingenio por causa de la necesidad. Un ejemplo fue el empleo de técnicas accesorias de protección de los caminos como la siembra de piñuela en las partes vulnerables cuando se hacían zanjas. ${ }^{47}$

\footnotetext{
${ }^{47}$ En 1854 en Santa María Ostuma se hizo "el zanjeado de chacras empiñando en las partes necesarias".
} 
Finalmente, con más recursos para contratar personal especializado, se empezaron a implementar nuevas técnicas europeas y a mediados del siglo se emitieron directrices locales más claras sobre construcción de caminos carreteros. ${ }^{48}$ Dos referentes técnicos, orientaban las intervenciones: el trazado y el firme. Se consideraba que los nuevos caminos abandonaran el trazado anterior con alineaciones rectas cuando el terreno lo permitía, con quiebres en ángulo y pequeños radios de giro, modificando el trazado cuando el tereno debía ascender a un puerto o descender al fondo de un valle. Abandonar la traza del camino anterior no significaba necesariamente el abandono de la franja territorial que recorría el camino ni los núcleos que comunicaba: se daba por entendido que las modificaciones venían determinadas por el nuevo papel del camino como ordenador o urbanizador del territorio que surcaba. En relación al firme, el modelo ideal era la conformación de calzadas con muros laterales que estuvieran bordeados por fosos, incluso en terreno llano, delimitando el camino. La tierra extraída de los fosos podía servir de pavimento al tiempo que rellenaba el espacio entre los dos muros laterales. Cuando el material no era de buena calidad, el espacio entre los muros debía rellenarse con piedras que disminuían en tamaño en la medida que se acercaran a la superficie. Al no conocer la naturaleza de los suelos y a falta de herramientas teóricas que en la actualidad proporciona la Mecánica de Suelos, las calzadas estaban expuestas a la improvisación.

Como fuera, se ejecutaron obras con prioridades definidas en torno a una primaria categorización de caminos de rueda, de herradura y vecinales, 1853. ${ }^{49}$ En Occidente, además del camino carretero que unía a Sonsonate con Acajutla (muy utilizado pero constantemente anegado debido a lo plano del terreno) fue importante el tramo entre Santa Ana y Sonsonate. Para 1862

\footnotetext{
${ }^{48}$ Se trata de un artículo tomado de la Gaceta de Guatemala y aparecido en la Gaceta del Gobierno del Salvador en la América Central, $N^{\circ}$ 102, Tomo 4 (Cojutepeque: 12 de abril de 1855), 4 .

${ }^{49}$ Decreto del 30 de marzo de 1853. Se mencionan aquí nada más los de rueda más sobresalientes.
} 
se completó el camino carretero y en 1867 se hicieron también carreteros tramos como el que salía de Santa Ana a Los Naranjos, Salcoatitán y Apaneca y a la villa de Ahuachapán, así como los los caminos que conectaban Sonsonate con Izalco, Nahulingo y Caluco, donde ya se hacía notar la importancia cafetalera. Otro camino de importancia fue el que conducía a Guatemala, aunque a finales de siglo aún contaba con tramos angostos. Para finales del siglo, se había hecho carretera la vía de Santa Ana a San Salvador (carretera “del Sur”) desde Coatepeque a Chalchuapa, la carretera de Sonsonate, cruzando la cordillera Apaneca-Lamatepec, y la "del Norte", que comunicaba a Santa Ana con Metapán vía Texistepeque. ${ }^{50}$ Para 1868, del camino Santa Ana - Sonsonate - Acajutla como del que conducía a la capital, "que antes solo era frecuentado por arrieros, hoy ruedan las carretas por todas partes, haciendo mas cómodo y económico al transporte de maderas \& a." se decía, agregando que estaba ya "tan generalizado el uso de las carretas, tanto en los caminos públicos, como en los que conducen á las haciendas y demas fincas, que se dificulta encontrar bestias, cuando se necesitan para equipajes". ${ }^{51}$

Debido a las dificultades que presentaba la ruta de acceso a la capital desde oriente ingresando por Santa Tecla (la cuesta del Guarumal), el camino más usual fue el que ingresaba desde el norte por Quezaltepeque, que se hizo carretero hasta aproximadamente 1882. El camino por El Guarumal, se hizo carretero hasta 1867 pero, finalizando el siglo continuaba dando serios problemas su mantenimiento.

El camino carretero más notable fue el de San Salvador- Santa Tecla. Su importancia se incrementó tras el terremoto que obligó a trasladar la capital a Santa Tecla, en que se hizo carretero. ${ }^{52}$ Desde entonces, un vínculo

\footnotetext{
${ }^{50}$ Guillermo J. Dawson, “Geografia Elemental de la República del Salvador”, Hachette y Cía (París: 1890; reedición del Grupo Agrisal, San Salvador: 2006), 15-20.

${ }^{51}$ República del Salvador, América Central, El Constitucional, № 63, Tomo 3 (San Salvador: 31 de diciembre de 1868), 4.

${ }^{52}$ Gaceta del Gobierno del Salvador en la América Central, № 81, Tomo 4 (Cojutepeque: 16 de noviembre de 1854), 2.
} 
estrecho quedó establecido entre ambas poblaciones y la comunicación vial se hizo tan frecuente que esta carretera de unos 12 kilómetros fue la primera en contar con un servicio regular de diligencias en la segunda mitad del siglo XIX.

En relación a la zona costera central, el camino San Salvador - Puerto de La Libertad como la ruta más atendida y el modelo de habilitación y mantenimiento de carreteras del siglo XIX. Para 1851 el camino no estaba completamente habilitado para carruajes, pero sí para los atajos de mulas. La situación cambió rápidamente y el año siguiente las mercaderías del primer barco llegado a La Libertad habían sido trasladadas en carretas. ${ }^{53}$ El camino, sin embargo, permaneció sometido a las inclemencias del tiempo hasta que en 1857 se mejoró en varios tramos y en 1864 se ejecutaron obras intensas para prestar mejores condiciones carreteras. Ese mismo año, se impuso para su conservación, ya como carretera, un primer reglamento de mantenimiento que se hizo extensivo a todo el país. En 1868 había un servicio establecido de diligencias que debió lidiar con los constantes daños y reparaciones que ameritó la vía.

La comunicación con el oriente del país siempre presentó grandes dificultades por la distancia, de manera que la habilitación carretera se fue ejecutando por tramos que irradiaban desde San Salvador o San Miguel. Desde los años 1840, el camino Cojutepeque - San Vicente llamó la atención con propósitos carreteros. Desde entonces se decía que el camino, que pasaba el río Jiboa, podía hacerse carretero pasando por la región de [San Rafael] Cedro [s] y Santo Domingo, con la disposición manifiesta de sus localidades por construirlo. Sin embargo, el tramo se hizo carretero hasta 1869. ${ }^{54}$ Para 1879 ya la carretera San Vicente- San Salvador, pasando por

\footnotetext{
${ }^{53}$ Gaceta del Gobierno del Salvador en la República de Centro América, No 47, Tomo 3 (San Salvador: 14 de mayo de 1852), 2.

${ }^{54}$ República del Salvador, América Central, El Constitucional, № 97, Tomo 3 (San Salvador: 2 de septiembre de 1869), 2.
} 
Cojutepeque, se mantenía con un tráfico importante, aunque el camino, ya carretero, ameritaba constantes reparaciones a finales del siglo. ${ }^{55}$ En el otro sentido, el camino San Vicente- San Miguel debió sufrir diversas intervenciones y mejoras entre 1849 y 1881 antes de ser completamente carretero. El paso del Lempa debió hacerse por barca todo el siglo.

Con un movimiento portuario más intenso, la región oriental captó particular interés para las autoridades. Esto orientó el desarrollo caminero en dos direcciones: al oeste, hacia La Unión y al sureste, hacia el puerto de La Concordia, en que cobró particular importancia Zacatecoluca. Con esta población como enclave, se trató de unir los puertos de La Unión y La Libertad en un proyecto visionario que no fructificó.

La zona Norte buscaba la conexión con el Pacífico a través de San Salvador. En Chalatenango confluían los caminos del norte del departamento, conectados por la necesidad, en sentido inverso, de mover productos a Honduras, hacia donde se conducía el camino que conducía a Guarita, pasando el Sumpul, sobre el que se planeó hacer un puente hasta que se construyó. Hacia el sur, el camino pasaba por Suchitoto, donde se entroncaba el de Ilobasco (aunque había otro de Ilobasco a Chalatenango) y debía sortear el paso por el caudaloso río Lempa, que se hacía con barcas. Para 1882, el camino Chalatenango- San Salvador era carretero, aunque ese año se ejecutaban importantes y nuevas reparaciones.

El aporte técnico más sobresaliente en la habilitación carretera vino de la introducción e implementación de la técnica del escocés John Loudon Mc Adam. Durante las tres primeras décadas del siglo XIX, tanto Mc Adam como Thomas Telford, así como un ingeniero de caminos francés, PierreMarie-Jerõme Trésaguet, habían perfeccionado en Europa los métodos y técnicas de construcción de carreteras. Mc Adam concebía la calzada como un

\footnotetext{
${ }^{55}$ República del Salvador, América Central, El Faro Salvadoreño, № 75, Tomo 2 (San Salvador: 28 de marzo de 1867), 3 .
} 
colchón de reparto de cargas de tráfico y sostenía que para que un camino soportara cualquier dcarga debía además estar bien drenado. En su sistema, una capa de piedra machacada, sin ningún elemento aglomerante se colocaba directamente sobre un cimiento de tierra que se elevaba del terreno circundante para asegurar un correcto desagüe. En su método se colocaban tres capas de piedra de unos diez centímetros de espesor cada una, compactadas primero a mano y luego apisonada con rodillos arrastrados por caballos. Con el apisonamiento y el peso del tráfico rodado, los bordes de las piedras se iban pulinedo, haciendo resbaladiza la superficie firme. Hasta que no aparecieron los vehículos con llantas de goma que levantaban la superficie de piedra y creaban baches o provocaban polvo, el macadam fue utilizado como la técnica más apropiada para los caminos en Europa y América en el siglo XIX.

El primer proyecto carretero de importancia que implementó las nuevas tecnologías tanto en firme como en trazado fue la ya referida carretera entre San Salvador y el Puerto de La Libertad. La presentación formal del proyecto incluyó detalles técnicos y logísticos como nunca se habían visto en las obras viales. La construcción del camino, que en realidad ya existía, pero que había que "arreglar", consideraba que su anchura en general fuera de ocho varas "pero en los lugares dificiles se limitará a la latitud necesaria" para que pudieran pasar dos carretas a la par. La forma del camino "será la mas conveniente, segun la calidad y circunstancias del terreno, procurando siempre la mayor duracion, las mas cortas distancias y salvando en lo posible las alturas, para que la ruta quede siempre aproximada al horizonte; y en los lugares bajos donde haya fangos, se harán calzadas para destruirlos, con sus correspondientes desagües a las orillas". ${ }^{56}$

Aunque referido a las ciudades, para 1867 ya se hablaba de la necesidad de que los empedrados fueran sustituidos por "macadan ó adoquines,

\footnotetext{
${ }^{56}$ Gaceta del Salvador, № 58, Tomo 6 (Cojutepeque: 14 de noviembre de 1857), 6 y 7.
} 
que no faltan en el pais", agregando que "todos sufren las molestias del empedrado actual, que se presta á aumentar el polvo en las calles; que causa ese estrepitoso ruido de las carretas, las cuales, ademas, se arruinan en poco tiempo; y que, con sus altos y bajos, molesta tanto á las personas que atraviesan de una acera á otra”. Una nueva era en la construcción carretera estaba iniciando y se acrecentaría con la República Cafetalera.

\section{Conclusiones}

La intensificación del movimiento marítimo no sólo facultó a los comerciantes salvadoreños la posibilidad de independizarse de sus iguales o mayores guatemaltecos: abrió el mundo a la nueva república. Con el tiempo, ese beneficio se acrecentó. Los acomodados pudieron viajar, salir a divertirse y educarse. Del extranjero llegaban noticias y costumbres. Se hablaba inglés, se bailaba polka. ${ }^{57}$ Aunque la bonanza en la economía salvadoreña no fue una constante, el tráfico marítimo facultó el acceso a nuevas oportunidades.

Una síntesis de las dificultades viales a solventar puede formularse como sigue. Primero, la carencia e inconstancia del financiamiento presupuestario y capitalista. Segundo, la debilidad política y administrativa del gobierno central y los municipios. Tercero, la ausencia de un plan general de equipamiento, alimentado por la debida información topográfica, además de concebido con una noción integradora. Cuarto, la inexistente o deficiente cantidad de personal capaz para dirigir y ejecutar las obras, así como de tecnología apropiada. Quinto, la ausencia de una política sistemática de mantenimiento.

Algunos esfuerzos permitieron compensar esas necesidades priorizando en la conexión entre los enclaves principales y los puertos, pero se dejaron de lado otras regiones y actores obviaron la función integradora de

\footnotetext{
${ }^{57}$ El Salvador Rejenerado, № 9, Tomo 2 (San Salvador, diciembre 17 de 1846), 35.
} 
los caminos. Lamentablemente con la misma visión, el impulso continuará gracias al fortalecimiento de economía cafetalera: con 128 carreteras que sumaban 575 leguas en todo el país en 1880; con la nueva carretera de San Vicente y Cojutepeque, que al pasar por el valle de Jiboa, acortaba la distancia en más de tres leguas; con la carretera de Verapaz que por la falda del volcán de San Vicente, conectaba con Zacatecoluca, de donde se comunicaba con los puertos de La Concordia y La Libertad y con la construcción de la carretera entre San Salvador y Santa Ana, por la cual ya circulaba, en 1887, la tercera parte de la producción de café. ${ }^{58}$

\footnotetext{
${ }^{58}$ Lindo Fuentes, refiere The American Annual Cyclopaedia, 1881, p. 805. DuPre, informe al Departamento de Estado, 28 de octubre de 1887. DUSC, 278.
} 


\section{REFERENCIAS BIBLIOGRÁFICAS}

\section{Periódicos}

Gaceta del Supremo Gobierno del Estado del Salvador, San Salvador. 1847-49 Gaceta del Gobierno del Salvador en la República de Centro América, Cojutepeque. 1854

República del Salvador, América Central El Salvador Rejenerado, San Salvador.1846-48

República del Salvador, América Central, El Faro salvadoreño, San Salvador. 1867

República del Salvador, América Central, El Constitucional, San Salvador.1868-69

\section{Libros}

Acosta, A. y Flores M. Municipio y Estado: La política municipal de los gobiernos de El Salvador hacia 1900 en "Poder, actores sociales y conflictividad. El Salvador, 1786-1972” López Bernal, Carlos Gregorio, compilador. San Salvador: Dirección Nacional de Investigaciones en Cultura y Arte de la Secretaría de Cultura de la Presidencia, 2011.

Dawson, G. Geografía Elemental de la República del Salvador. Hachette y Cía., París, 1890. San Salvador: Grupo Agrisal, 2006.

Gutiérrez y Ulloa, A. Estado General de la provincia de San Salvador, Reyno de Guatemala, presentado por el intendente Antonio Gutiérrez y Ulloa en el año de 1807. San Salvador: Dirección General de Publicaciones, Ministerio de Educación, 1966

Lindo, Héctor. La Economía de El Salvador en el siglo XIX. San Salvador: Dirección de Publicaciones e Impresos, Consejo Nacional para la Cultura y el Arte, CONCULTURA, 2002 
Menéndez, I. Recopilación de las Leyes del Salvador, en Centro-América. Tomo II. Guatemala, 1885

Squier, E. Apuntamientos sobre Centroamérica. Honduras y El Salvador. Traducción de León Alvarado. Notas actualizadas de William V. Davidson. Colección Cultural de Centroamérica Serie Viajeros no. 5. Managua : Fundación Vida, 2001

Pérez M. Los caminos reales de América en la legislación y en la historia. Sevilla: Universidad de Sevilla. Anuario de Estudios Americanos, Tomo LVIII, I, 2002 